This item was submitted to Loughborough's Research Repository by the author.

Items in Figshare are protected by copyright, with all rights reserved, unless otherwise indicated.

\title{
Analysis of key performance parameter extraction from current voltage measurements of photovoltaic devices
}

PLEASE CITE THE PUBLISHED VERSION

http://www.ieee-pvsc.org/PVSC37/

PUBLISHER

(C) IEEE

VERSION

AM (Accepted Manuscript)

LICENCE

CC BY-NC-ND 4.0

\section{REPOSITORY RECORD}

Qasem, Hassan, Thomas R. Betts, Ira D. Sara, Martin Bliss, Jiang Zhu, and Ralph Gottschalg. 2019. "Analysis of Key Performance Parameter Extraction from Current Voltage Measurements of Photovoltaic Devices". figshare. https://hdl.handle.net/2134/8462. 
This item was submitted to Loughborough's Institutional Repository (https://dspace.lboro.ac.uk/) by the author and is made available under the following Creative Commons Licence conditions.

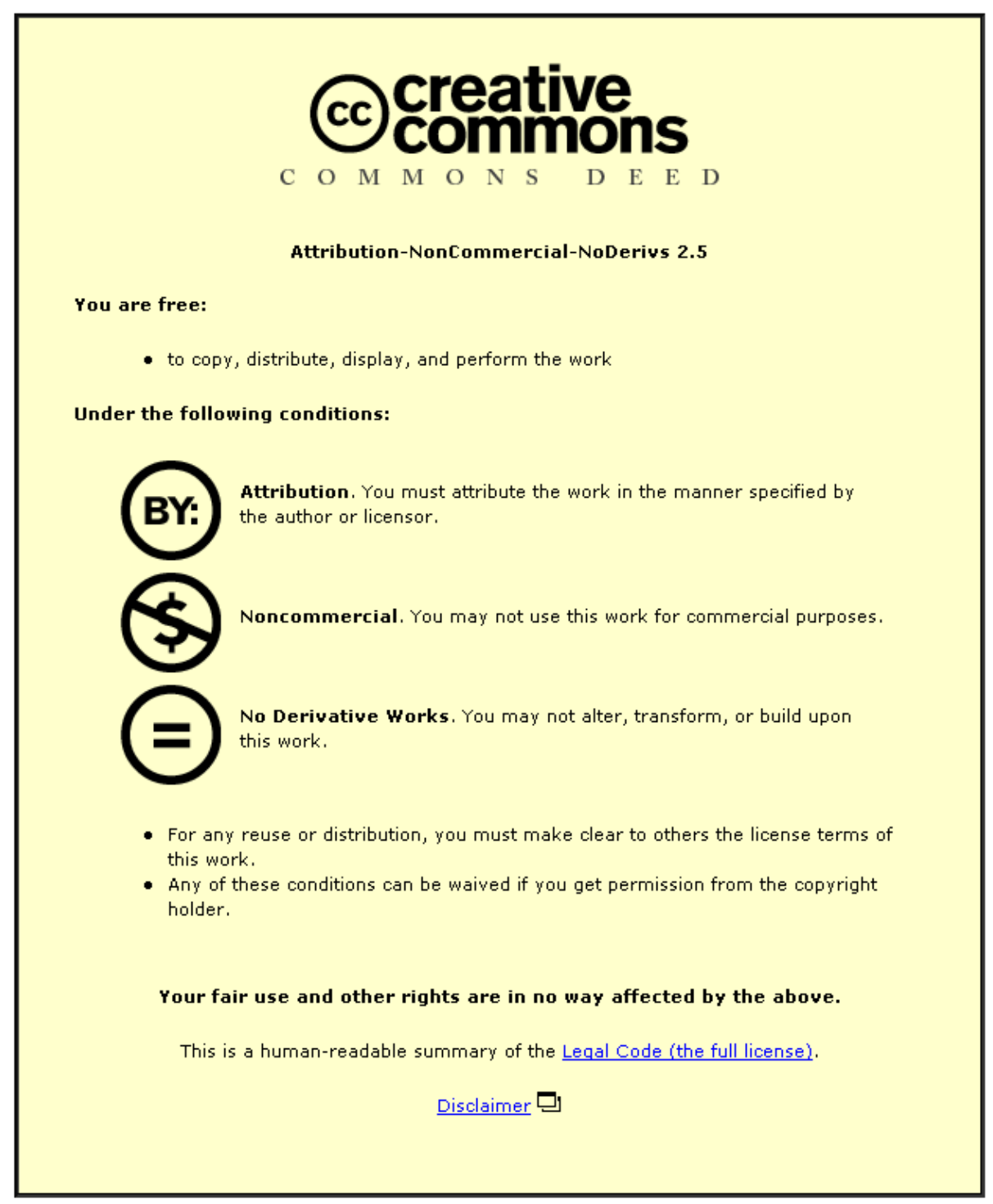

For the full text of this licence, please go to: http://creativecommons.org/licenses/by-nc-nd/2.5/ 


\title{
ANALYSIS OF KEY PERFORMANCE PARAMETER EXTRACTION FROM CURRENT VOLTAGE MEASUREMENTS OF PHOTOVOLTAIC DEVICES
}

\author{
H. Qasem*, T.R. Betts, I.D. Sara, M. Bliss, J. Zhu and R. Gottschalg. \\ Centre for Renewable Energy Systems Technology (CREST), Department of Electronic and Electrical \\ Engineering, Loughborough University, Leicestershire, LE11 3TU, UK. \\ *Phone: +44(0)1509 63 5337, Fax: +44(0)1509 635301 E-mail: H.Qasem@lboro.ac.uk
}

\begin{abstract}
The accuracy of the characterisation of photovoltaic devices may be affected by the method of extraction of the performance parameters. This paper investigates potential uncertainties in extracted short circuit current $\left(\mathrm{I}_{\mathrm{sc}}\right)$, open circuit voltage $\left(\mathrm{V}_{\mathrm{oc}}\right)$ and maximum power $\left(P_{\mathrm{mpp}}\right)$ from current-voltage $(\mathrm{I}-\mathrm{V})$ data of a photovoltaic (PV) device. Different interpolation methods are compared. Linear interpolation, straight line regression and polynomial regression methods are compared in different scenarios in which the curve point density and point selection for extraction are varied. The comparison shows that regression approaches can produce more accurate results when appropriate curve point density and point selection are selected. When attempting to extract $I_{s c}$. $V_{o c}$ and $P_{m p p}$ from noisy or irregular I-V data, linear interpolation is less robust than regression because of the irregular point distribution on the I-V curve.
\end{abstract}

\section{INTRODUCTION}

The increase in demand for PV applications requires more accurate and reliable methods of determining PV device ratings. While the accuracy of measuring device power varies when different equipment is used, this is understandable due to the different uncertainty associated with every measuring device [1-2]. Analysis of the measured raw I-V data to yield key performance parameters should introduce minimal uncertainties; however this is not necessarily the case due to a lack of standardization. The extraction of the short circuit current $\left(I_{\mathrm{sc}}\right)$, open circuit voltage $\left(\mathrm{V}_{\mathrm{oc}}\right)$ and maximum power point $\left(\mathrm{P}_{\mathrm{mpp}}\right)$ can thus lead to different results when the same device measurement data is analyzed with different methods.

When a PV device's I-V curve is measured, the number of measured points and the selection of the start and end points can introduce different results when $V_{o c}, I_{s c}$ and $P_{m p p}$ are extracted [3], introducing uncertainty in the final results. This problem worsens when measurement noise is also considered (Figure 1). Additional electronic components used in some PV modules, such as blocking and bypass diodes, can introduce further complications to parameter extraction when I-V data are measured with a voltage sweep that extends to reverse bias (Figure 2 and Figure 3 ) or forward currents, this issue was also mentioned by Piliougine et al. as a problem that introduces more uncertainty to the extraction of I-V data main parameters [4]. This requires special considerations to avoid distortion of the extracted key performance parameters, particularly $I_{s c}$. I-V curves measured with passive loads can limit the minimum voltage attained on the sweep (Figure 4), with additional parameter extraction uncertainty depending on the method employed.

Very little detail is presented in most of the standards in how to extract $I_{s c}, V_{o c}$ and $P_{\text {mpp }}$ from the STC measured I-V data. ASTM E948 has the most description as it recommends interpolation or extrapolation for $V_{o c}$ within $\pm 0.005 \mathrm{~V}$ from zero current and $\mathrm{ISC}_{\mathrm{SC}}$ within $\pm 0.001 \mathrm{~A}$ from zero voltage and the use of a fourth-order polynomial least square fit for extracting $P_{m p p}[2]$.

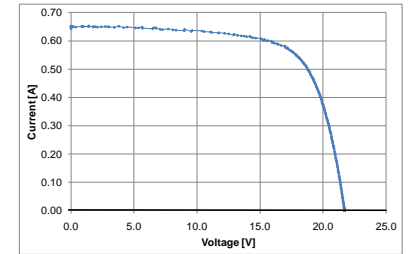

Figure 1: Measurement noise effect on I-V curve (measurement taken in commercial simulator)

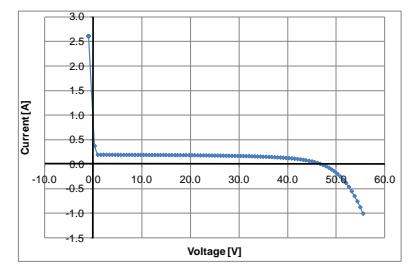

Figure 3: Bypass diode effect in reverse I-V sweep (measurement taken in outdoor system).

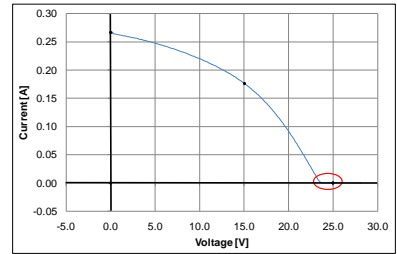

Figure 2: Blocking diode effect in battery charger PV modules. (measurement taken in commercial solar simulator)

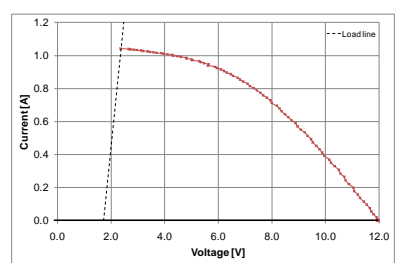

Figure 4: Limiting voltage effect of passive load sweep (measurement taken in experimental outdoor system)
On the other hand, IEC61853-1 recommends linear regression for fitting both $V_{o c}$, $I_{s c}$ and a 'polynomial' fit 
for obtaining $P_{m p p}[5]$. It does not specify the range of points or accuracy recommended for obtaining the extracted parameters. Other papers recommend a range of points where the voltage is $10 \%$ or less of $V_{\text {oc }}$ and current $12 \%$ or less of $I_{s c}[6]$. Emery suggests that the closest two points bracketing the $V_{o c}$ or $I_{s c}$ are to be used to apply linear regression to obtain the $V_{o c}$ or $I_{s c}$ values. He also stated that increasing the number of points surrounding the extracted values can reduce the uncertainty of the extracted value [7].

This paper focuses on investigating the effect of extracting $V_{o c}, I_{s c}$ and $P_{m p p}$ from an I-V curve under the variation of the total number of points on the curve and the number of points used for the parameter extractions with linear interpolation, straight line and polynomial regression [3].

\section{SHORT CIRCUIT CURRENT, OPEN CIRCUIT VOLTAGE AND MAXIMUM POWER EXTRACTION}

Extracting $I_{s c}$ and $V_{o c}$ from an I-V curve measurement are generally more complex than selecting the device current at zero voltage, and device voltage at zero current [1]. This is because in most cases there are not specific measurements at these exact zero points.

One method for obtaining $V_{o c}$ and $I_{s c}$ is by selecting a number of measured points that bracket the zero voltage or current, and then interpolating or extrapolating to the zero point [2][8]. The other approach is via fitting a line through selected points which bound the zero voltage or current to get the value at the zero point [9]. A similar approach may be used to obtain the maximum power point by using a second or higher order polynomial, or other model, fitting to either the current-voltage or the derived power-voltage measurement points [10].

A mono-crystalline calibrated module was used as the basis of the investigation. The module was measured and the parameters were extracted. Simulated I-V curves were used, to generate idealised, unbiased 'measurements'. The module was simulated using the one diode model with PSPICE, with input model parameters extracted from an I-V curve measured at Standard Test Conditions (STC).

$$
I(V)=I_{0}\left(e^{\frac{q\left(V-I R_{s}\right)}{n K T}}-1\right)+\frac{V-I R_{s}}{R_{s h}}-I_{p h}
$$

A series of I-V curves were then generated with PSPICE with different numbers of points on the curve. The boundaries of voltage in the simulation were selected to provide some points into the reverse bias region and also beyond open circuit.
The robustness of installation is investigated by adding a simulated Gaussian noise. Special cases involving additional electrical components were simulated using PSPICE.

Code was written to extract $V_{o c}, I_{s c}$ and $P_{\text {mpp }}$ from the curves, with fitting methods implemented in Delphi and interpolation in MATLAB. Straight line regression was applied to the simulated data by fitting a line on a selected number of points close about the zero circuit and zero voltage points. A second-order polynomial model was also tried for determining $\mathrm{V}_{\mathrm{oc}}$ (the $\mathrm{I}_{\mathrm{sc}}$ result from the straight line fit could not be improved upon). The points about the peak on the power-voltage curve were fitted with a second order polynomial and a third order polynomial, to find the $P_{\text {mpp }}$. The linear interpolation uses the existing points on the curve to interpolate to the desired point.

\section{RESULTS AND DISCUSSION}

\section{A. Linear interpolation}

Different simulation where carried out with varying the number of points on the curve from 10 points to 1000 points. The point selection for $I_{s c}$ and $V_{o c}$ varied from 2 points up to 50 points. The extracted $V_{o c}$ and $\mathrm{I}_{\mathrm{sc}}$ are shown in Figure 5 and Figure 6. The maximum deviation from the ideal values of $\mathrm{I}_{\mathrm{sc}}$ and $\mathrm{V}_{\text {oc }}$ are $0.04 \%$ and $0.18 \%$.

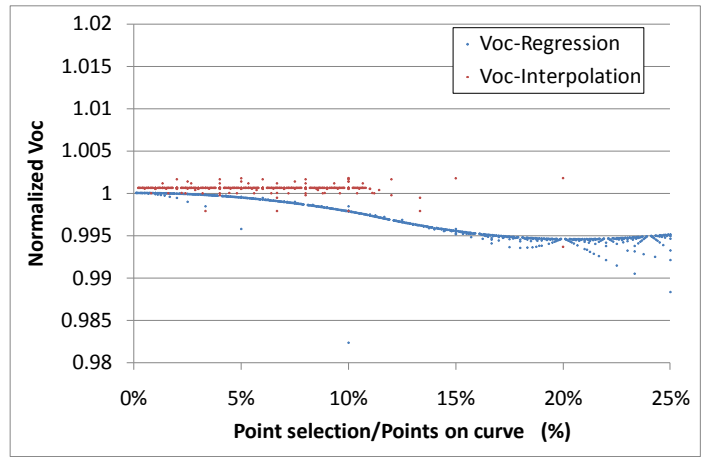

Figure 5: Normalized $\mathrm{V}_{\text {oc }}$ with respect to the simulated value extracted with linear regression and interpolation.

Linear interpolation showed less deviation between the selected points when higher numbers of points are selected to perform the calculations. The optimal point selection to curve ratio that can be used is $11 \%$ to interpolate the value of $\mathrm{V}_{\text {oc }}$ (see Figure 5). On the other hand, $I_{s c}$ is much easier to extract because the region near $I_{s c}$ value tends to be less curved than that of $\mathrm{V}_{\mathrm{oc}}$ and thus the optimum point selection to curve ratio needed is $20 \%$, as shown in Figure 6 . It is also noted that by using linear interpolation an inherent average error was noticed within the 
extracted values of $\mathrm{I}_{\mathrm{sc}}$ of $-0.017 \%$ and $0.064 \%$ for all $\mathrm{V}_{\text {oc }}$ values.

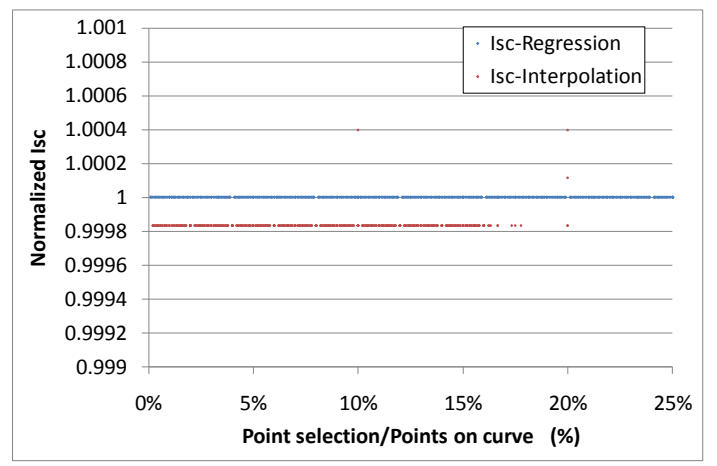

Figure 6: Normalized $I_{s c}$ values with respect to the simulated values extracted with linear regeression and interpolation

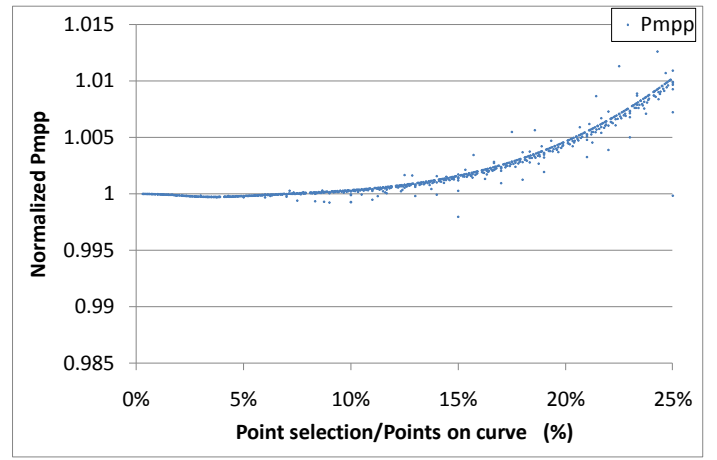

Figure 7: Normalaized $\mathrm{P}_{\mathrm{mpp}}$ values with respect to the simulated values, extraxted by $2^{\text {nd }}$ order pylonomal regression.

\section{B. Straight line and polynomial regression}

The maximum deviation from the simulated I-V values for $I_{\mathrm{sc}}, \mathrm{V}_{\mathrm{oc}}$ and $\mathrm{P}_{\mathrm{mpp}}$ that where calculated with regressions are $11.3 \%, 1.0 \%$ and $1.1 \%$. The large deviations may discourage the use of regression to extract the key values from I-V data because they can introduce error in the calculation of $\mathrm{I}_{\mathrm{sc}}, \mathrm{V}_{\mathrm{oc}}$ and $I_{m p p}$, which is not true if used correctly. Linear regression showed more accurate but less stable results than the result calculated from linear interpolation, mainly because no inherent error was found within the calculation. Regression eliminates the error by fitting a linear equation on the selected points, then using it to solve for the desired zero values, which means point selection and curve density will mainly affect the curve fitting. This approach makes the linear regression method less prone to measurement noise and other external factors.

The effect of point selection and curve density on $I_{s c}$ values extracted with regression and interpolation is shown in Figure 6. The maximum optimal point selection to curve ratio is up to $25 \%$ for extraction of $\mathrm{I}_{\mathrm{sc}}$. For $\mathrm{V}_{\mathrm{oc}}$ the dominating factor is the point selection, see Figure 5 . The maximum point selection to curve ratio is up to $2 \%$; this behaviour can be explained by the relation of fitted line and the slope at the I-V curve. When higher numbers of points are used to fit the $\mathrm{I}_{\mathrm{sc}}$ or $\mathrm{V}_{\mathrm{oc}}$ regions of the $\mathrm{I}-\mathrm{V}$ data the effect of slope curve becomes more apparent on the fitted curve, and thus affect the calculated accuracy when regression is used.

The previous problem with the slope is reduced when $P_{m p p}$ is extracted (Figure 7). Mainly because a second order polynomial curve is fitted on the selected points around the maximum power point of the measured I-V data. Since linear interpolation produces a significant error when used to extract the $\mathrm{P}_{\mathrm{mpp}}$, only polynomial regression was used. When a polynomial curve was fitted, a smoother curve showed better results, with the optimal point selection to curve point ratio found to be $2 \%$.

\section{Other extraction models}

Different extraction methods where used to extract $\mathrm{V}_{\mathrm{oc}}, \mathrm{I}_{\mathrm{sc}}$ and $\mathrm{P}_{\mathrm{mpp}}$ from a normal $\mathrm{I}-\mathrm{V}$ curve where the curve number of points is fixed at 300 points. In addition to the chosen linear interpolation and regression cubic and spline interpolation were used to extract $\mathrm{I}_{\mathrm{sc}}$ and $\mathrm{V}_{\mathrm{oc}}$, quadratic regression for extracting $\mathrm{V}_{\mathrm{oc}}$ and cubic regression for the extraction of $P_{\text {mpp }}$.

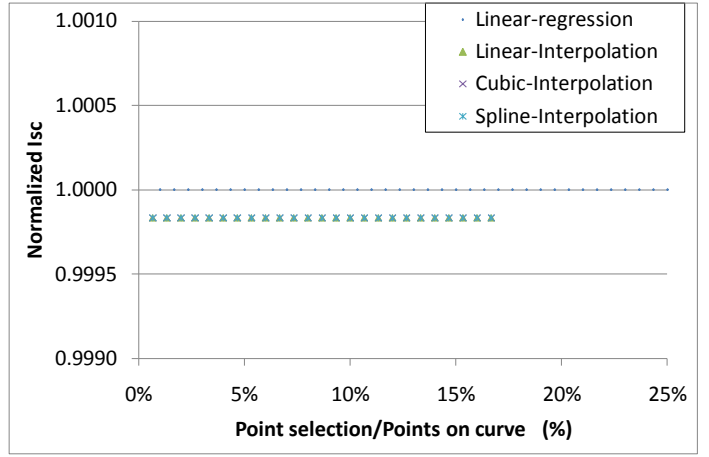

Figure 8: Different methods applied to extract $I_{s c}$ values from the modelled I-V data with the number of points on the curve fixed at 300 .

$\mathrm{n}$ the case of $\mathrm{I}_{\mathrm{sc}}$, the other types of extraction did not introduce any improvements from the values extracted with linear regression (Figure 8). As for $\mathrm{V}_{\mathrm{oc}}$ quadratic fitting and cubic fitting for $P_{\text {mpp }}$ showed better results at higher point selected to point on curve ratio, see Figure 9 and Figure 10. 


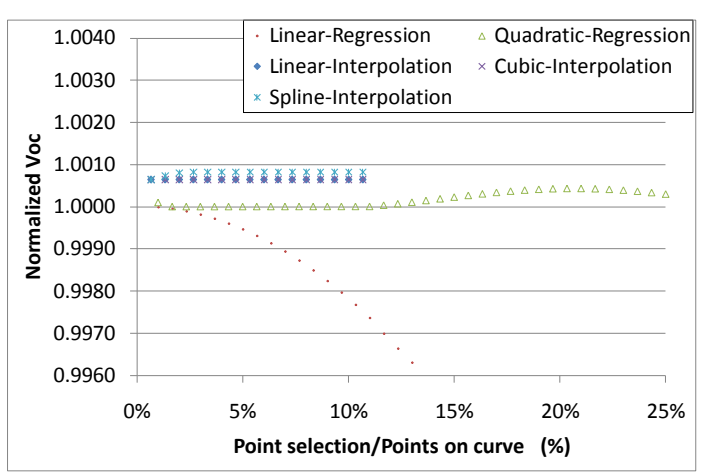

Figure 9: Different methods applied to extract $V_{o c}$ values from the modelled I-V data with the number of points on the curve fixed at 300 .

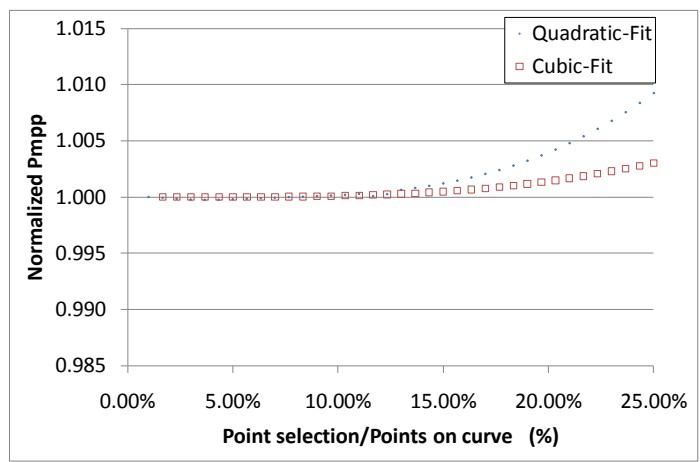

Figure 10: Quadratic and cubic fitting is used to extract the $P_{\text {mpp }}$ values. Again the curve number of points is fixed at 300.

\section{Blocking diode}

In some modules, a series blocking diode is utilized to prevent reverse current to flow back into the module. The behaviour of such a device was modelled with PSPICE and then the main parameters were extracted using linear interpolation and regression as shown in Figure 11.

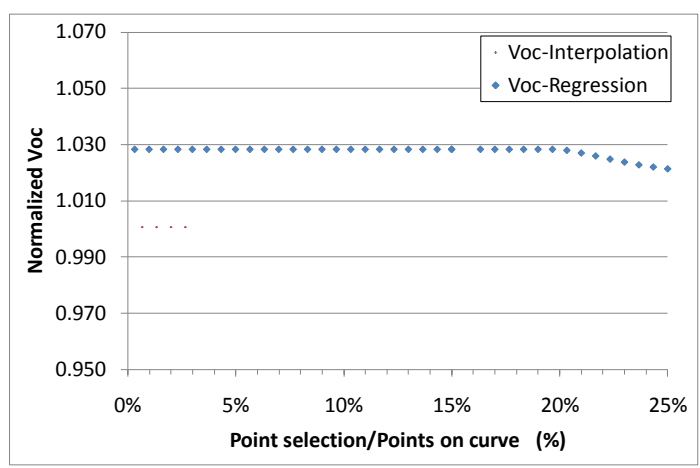

Figure 11: $\mathrm{V}_{\mathrm{oc}}$ values extracted with linear regression and linear interpolation for a module with a blocking diode.

The problematic region in this type of measured I-V data is around the $V_{\text {oc }}$ because the current will be very small and will not cross to zero. This makes extracting the $V_{o c}$ less accurate with regression and more accurate with linear interpolation due to less accurate fitting of the straight line although only few points can be selected to enable linear interpolation as shown in Figure 11. On the other hand, an early detection of this type of distortion can allow better fitting as the distorted points can be identified and avoided to allow a better extraction of the parameters with regression.

\section{E. Bypass diode in reverse $I-V$ sweep}

This problem happens when a PV module with a bypass diode is measured with a voltage sweep that extends to reverse bias, as shown in Figure 3 . This effect can vary with different devices measured with different tracers. Mainly because it limits the number of points that can be selected around the $I_{\mathrm{sc}}$ value. Figure 12 shows the $I_{\mathrm{sc}}$ extracted from a simulated I$\checkmark$ curve with a bypass diode and a fixed number of curve points of 300 . At a point selected to points-oncurve ratio of $16 \%$, straight line regression becomes less accurate than linear interpolation. This is mainly due to the less accurate fitting of the straight line due to the effect of the bypass diode.

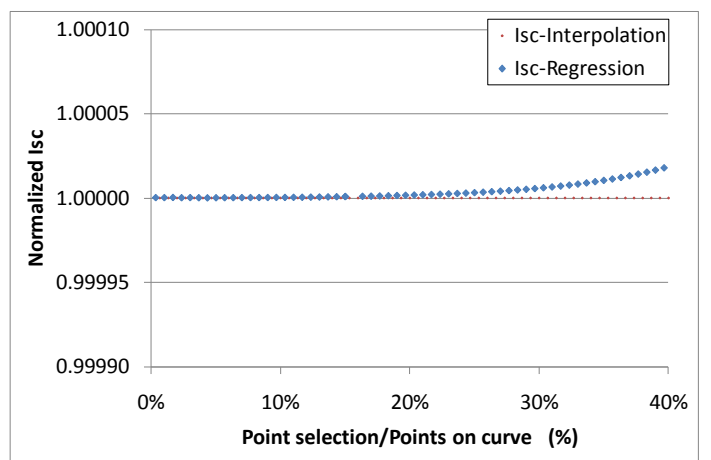

Figure 12: Effect of bypass diode on the extraction of $\mathrm{I}_{\mathrm{sc}}$ values.

\section{F. Limiting voltage effect on passive load sweep} This effect is shown in I-V tracers with a passive type of load, which is mainly used for outdoor measurements. The initial voltage was limited and thus provided an I-V curve with missing points around the $\mathrm{I}_{\mathrm{sc}}$ as shown in Figure 4. Due to the missing points, using linear interpolation was not possible, instead only the closest points were used to extrapolate to the $I_{s c}$ value. Straight line regression provided a better result for extracting $I_{s c}$ with selecting point selection to point on curve ratio of $5 \%$ or higher (Figure 13), nevertheless this can introduce a major problem for other technologies or modules with less linear region around $\mathrm{I}_{\mathrm{sc}}$. 


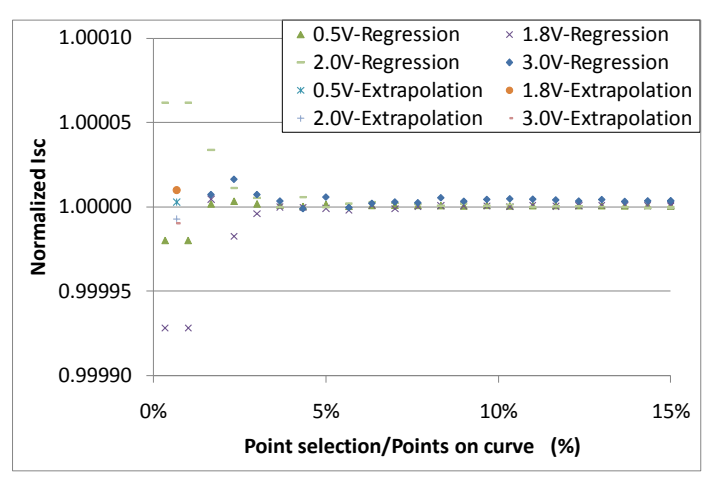

Figure 13: Extracted $\mathrm{I}_{\mathrm{sc}}$ for $\mathrm{PV}$ module measured with a passive load sweep with initial voltage limited to $0.5 \mathrm{~V}, 1.8 \mathrm{~V}, 2 \mathrm{~V}$ and $3 \mathrm{~V}$. The curve number of points is fixed at 300 points.

\section{G. Measurement noise}

Gaussian noise levels of $0.1 \%, 0.2 \%, 0.5 \%$ and $1.0 \%$ where added to the curve of 300 points, keeping in mind that acceptable error level in electronic components is in the range of $0.2 \%$ [1], which means that noise level should be less than the $0.2 \%$ value. Regression and linear interpolation were used on the curves to extract the desired parameters. For $\mathrm{I}_{\mathrm{sc}}$ and $\mathrm{V}_{\mathrm{oc}}$, linear regression showed better results when compared to the linear interpolation, see Figure 14-Figure 16. This again can be attributed to the fact that interpolation uses the actual noisy I-V data to interpolate for the zero point, while with regression a fitted linear equation will be used instead.

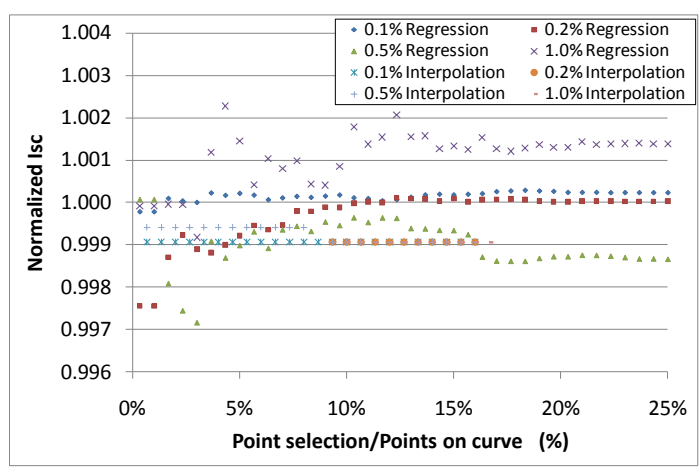

Figure 14: $I_{s c}$ extracted for a I-V curve with different measurement noise levels being added.

Noise is a random feature, which will have different results each time the process is carried out. To minimise this, a Monto-Carlo simulation was used to assess the uncertainties related to parameter extraction of the $\mathrm{I}_{\mathrm{sc}}, \mathrm{V}_{\mathrm{oc}}$ and $\mathrm{P}_{\mathrm{mpp}}$ [11]. Two levels of noise, $0.2 \%$ and $1.0 \%$, were Monte-Carlo simulated and applied to both voltage and current values of the simulated I-V data. The $\mathrm{I}_{\mathrm{sc}}, \mathrm{V}_{\mathrm{oc}}$ and $\mathrm{P}_{\mathrm{mpp}}$ values were then extracted with straight line and quadratic regression, limiting the curve number of points to 300 , and varying the selected number of points up to 50. This procedure was repeated 10,000 times and the results are presented in Table 1 where it shows that uncertainties in of extracting PV device main parameters are higher with higher level of noise. Also with a noise level of $0.2 \%$ an average standard deviation of $0.05 \%$ is to be expected for $\mathrm{I}_{\mathrm{sc}}$ and $\mathrm{V}_{\mathrm{oc}}$ values, and $1.8 \%$ for the extraction of $P_{m p p}$.

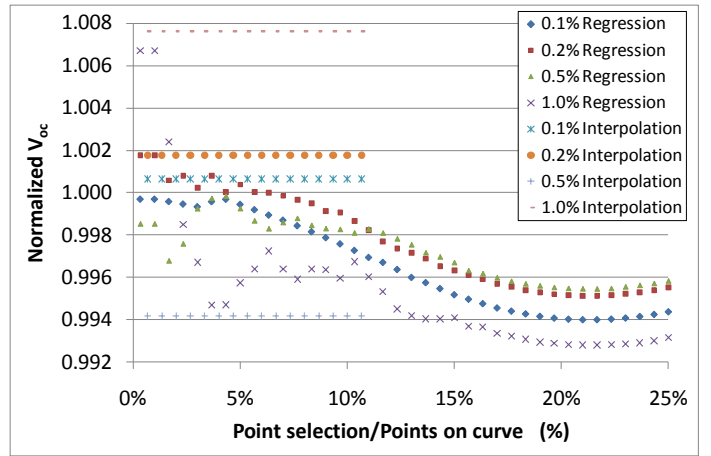

Figure 15: $\mathrm{V}_{\text {oc }}$ extracted for a I-V curve with different measurement noise levels being added.

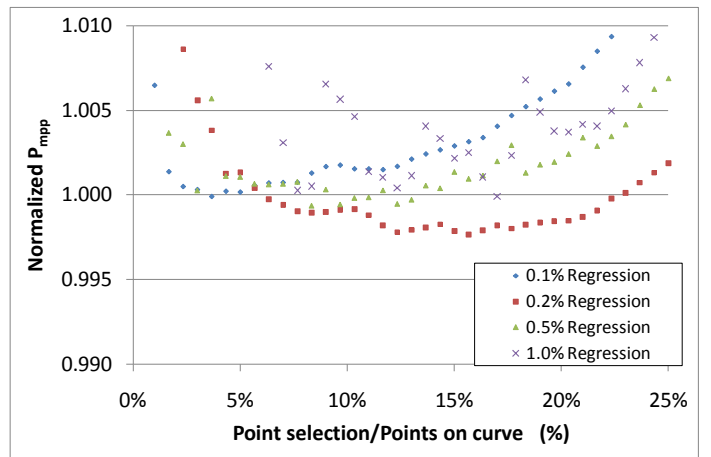

Figure 16: $P_{m p p}$ extracted for a I-V curve with different measurement noise levels being added.

Table 1: Extracted normalized parameters for noisy $\mathrm{I}-\mathrm{V}$ data, where $\mathrm{I}_{\mathrm{sc}}$ and $\mathrm{V}_{\mathrm{oc}}$, are extracted 10,000 times with linear regression and $P_{m p p}$ with quadratic regression.

\begin{tabular}{lcccccc}
\hline & $\begin{array}{c}\mathbf{I}_{\text {sc }} \\
\text { Mean }\end{array}$ & $\begin{array}{c}\mathbf{I}_{\text {sc }} \\
\text { StDev }\end{array}$ & $\begin{array}{c}\mathbf{V}_{\text {oc }} \\
\text { Mean }\end{array}$ & $\begin{array}{c}\mathbf{V}_{\text {oc }} \\
\text { StDev }\end{array}$ & $\begin{array}{c}\mathbf{P}_{\text {mpp }} \\
\text { Mean }\end{array}$ & $\begin{array}{c}\mathbf{P}_{\text {mpp }} \\
\text { StDev }\end{array}$ \\
\hline Max & 1.0000 & 0.0012 & 1.0000 & 0.0012 & 1.0225 & 0.2839 \\
Min & 1.0000 & 0.0003 & 0.9948 & 0.0003 & 0.9996 & 0.0011 \\
Avg & 1.0000 & 0.0005 & 0.9979 & 0.0005 & 1.0015 & 0.0179 \\
\hline \multicolumn{7}{c}{$\mathbf{1 . 0 \%}$ Noise } \\
\hline Max & 0.9989 & 0.0828 & 1.0000 & 0.0062 & 1.4354 & 0.5777 \\
Min & 0.9903 & 0.0438 & 0.9948 & 0.0016 & 0.9943 & 0.0455 \\
Avg & 0.9980 & 0.0476 & 0.9979 & 0.0024 & 1.0176 & 0.9095 \\
\hline
\end{tabular}




\section{CONCLUSION}

When $I_{s c}, V_{o c}$ and $P_{m p p}$ data are extracted from a measured I-V curve, different accuracy for the results can be observed depending on the point density of the measured I-V data and on the selected points over which the extractions are preformed. Extracting the parameter with Interpolation requires less calculation and thus it proved to be faster to use when less accuracy are acceptable. On the other hand, the vulnerability of interpolation to noise may produce less robust results in realistic measurement data, which makes linear regression the better and more robust choice.

Different types of interpolations and regression were explored in this paper, where for a standard I-V curve, straight line regression proved to present slightly better results than linear regression. When the right numbers of points are selected for the I-V measured data and the right range of points is selected for the curve fitting, extracting data with regression can be the most reliable method. Considering the different requirements for $I_{s c}, V_{o c}$ and $P_{\text {mpp }}$, the optimal point selection to curve point ratio were found to be up to $25 \%$ for $I_{s c}$, up to $2 \%$ for both $\mathrm{V}_{\mathrm{oc}}$ and $\mathrm{P}_{\mathrm{mpp}}$.

Special case I-V curves can be problematic when an $\mathrm{I}_{\mathrm{sc}}, \mathrm{V}_{\mathrm{oc}}$ and $\mathrm{P}_{\mathrm{mpp}}$ are to be extracted. Blocking diodes can increase the error in using regression and reduce the possible number of points that can be used to interpolate to the $V_{o c}$ value. On the other hand bypass diodes are less problematic in the term of extracting $\mathrm{I}_{\mathrm{sc}}$, where the bypass diode effect is present. That is assuming that enough number of points is presented around the $I_{s c}$ value. Limiting voltage effect introduced by passive type of I-V tracers are better measured with straight line regression as not enough number of points is there to apply linear interpolation.

A current voltage data with a noise level of $0.2 \%$ or less can be extracted with the use of straight line regression although an average standard deviation of $0.05 \%$ is to be expected for the extraction of $I_{s c}$ and $V_{o c}$ and $1.8 \%$ for the extraction of $P_{m p p}$.

\section{REFERENCES}

[1] Measurement of photovoltaic current-voltage characteristics, IEC Standard, 60904-1, 2006.

[2] Standard test method for electrical performance of photovoltaic cells using reference cells under simulated sunlight, ASTM Standard, E948-09, 2009.

[3] R. Gottschalg, "Environmental Influences on the performance of thin film solar cells," Ph.D. dissertation, Dept. of Electron. and Elect. Eng., Loughborough Univ., United Kingdom, 2001.

[4] M. Piliougine, J. Carretero, L. Mora-López and M. Sidrach-de-Cardona, "Experimental system for current-voltage curve measurement of photovoltaic modules under outdoor conditions," Progress in. Photovoltaics: Res. Appl., Jan. 2011.

[5] Photovoltaic (PV) module performance testing and energy rating, IEC Standard, 61853-1, 2011.

[6] J. A. del Cueto and T. J. McMahon, "Performance of single-junction a-si modules under varying conditions in the field," in Twenty-Sixth IEEE Photovoltaic Specialists Conference, Anaheim, CA., 1997, pp. 1205-1208.

[7] K. Emery, "Measurement and Characterization of Solar Cells and Modules," pp. 701-747, 2010.

[8] J. C. H. Phang, D. S. H. Chan and J. R. Phillips, "Accurate Analytical Method for the Extraction of Solar-Cell Model Parameters," Electron. Lett., vol. 20, pp. 406-408, 1984.

[9] D. S. H. Chan, J. R. Phillips and J. C. H. Phang, "A comparative study of extraction methods for solar cell model parameters," Solid-State Electronics, vol. 29, pp. 329-337, 3, 1986.

[10] M. C. Di Piazza, A. Ragusa and G. Vitale, "Identification of photovoltaic array model parameters by robust linear regression methods," in International Conf. on Renewable Energies and Power quality, Valencia, Spain. 2009.

[11] Uncertainty of Measurement. Part 3, Guide to the Expression of Uncertainty in Measurement (GUM:1995). Supplement 1, Propagation of Distributions using a Monte Carlo Method. Geneva : International Organisation for Standardization, JCGM, 101, 2008. 